\title{
CRATERS PRODUCED BY UNDERGROUND EXPLOSIONS
}

\author{
Bibiana M. Luccioni ${ }^{\text {a,c }}$ and Ricardo D. Ambrosini ${ }^{\text {b,c }}$ \\ ${ }^{a}$ Instituto de Estructuras, Universidad Nacional de Tucumán, Av. Roca 1800, 4000 S.M. de Tucumán, \\ Argentina,labest@herrera.unt.edu.ar.edu.ar,www.herrera.unt.edu.ar/iest \\ ${ }^{b}$ Facultad de Ingeniería, Universidad Nacional de Cuyo \\ Centro Universitario - Parque Gral. San Martín - 5500 Mendoza, dambrosini@uncu.edu.ar , \\ http://fing.uncu.edu.ar/
}

Keywords: Crater, blast load, soil, hydrocode

\begin{abstract}
Extensive research activities in the field of blast loads have taken place in the last few decades. There are many experimental results related to underground explosions. The mechanism of crater formation is complex and it is related to the dynamic physical properties of air, soil and soil-air interface. Studies concerned with the characteristics of craters caused by explosions usually resort to dimensional analysis and statistics. Some empirical equations proposed for the evaluation of crater dimensions can be found in the literature. Nevertheless, they were obtained for particular type of soils, shapes of explosives, ranges of explosive mass and depth of explosive and they present considerable variability.

The main objective of this paper is to prove the accuracy of numerical simulation of craters produced by underground explosions. For this purpose, the numerical analysis of crater formation due to underground explosions is performed with a hydrocode. Several numerical approaches are carried out using different models and processors for the soil. Moreover, different alternatives for the constitutive model of the soil are used.

In order to validate the numerical approach and prove its ability to model the crater formation, comparison with experimental results is performed. Many simulations of the same physical model lead to the same crater dimensions and a good agreement between the test results and the predicted crater measures is achieved.
\end{abstract}




\section{INTRODUCTION}

Tests of crater formation are appropriate tools to study the blast phenomena, the behavior and destructive power of different explosives and the response of soils and rocks under this type of load (Persson et al. 1994). The mechanism of crater formation is complex and it is related to the dynamic physical properties of air, soil and soil-air interface. Even very carefully performed cratering tests give deviations in the dimensions measured of about $10 \%$, while differences of as much as $30 \%$ to $40 \%$ are common (Bull et al. 1998)

A cavity is always formed when a confined explosion is produced in a mass of soil. If the explosion is close to the surface, a crater is formed, a complex interaction taking place between gravity effects, soil strength and transient load conditions. The most important variables in defining the crater shape and size are the mass $\mathrm{W}$ of the explosive and the depth of the detonation beneath the air/soil interface $d$. When $d<0$, the explosive is detonated over the air/soil interface, $d=0$ when the detonation occurs in the air/soil interface and $d>0$ when the explosive is detonated beneath the soil surface. For $\mathrm{d}>0$, the crater mechanism is altered by gravitational effects. When the depth of the detonation increases, larger amounts of subsoil must be expelled by the explosion. Thus the crater radius and the depth of the crater increase when $d$ increases, until a certain limit value, from which they rapidly decrease (Bull et al. 1998).

Studies concerned with the characteristics of craters caused by explosions usually resort to dimensional analysis and statistics. The scaling law establishes that any linear dimension " $L "$ of the crater can be expressed as a constant multiplied by $\mathrm{W}^{\alpha}$ divided by the distance of the charge from the ground, where W represents the equivalent TNT mass of explosive and $\alpha$ is a coefficient depending on if the gravitational effects can be neglected or not. In the first case the cubic root law is applicable $(\alpha=0.33)$ and in the other cases the functional dependence can be quite complex.

Baker et al. (1991) presents a dimensional study to model the crater formation phenomenon in the case of underground explosions. Six parameters are chosen to define the problem: the explosive mass $\mathrm{W}$, the depth of the explosive charge, $\mathrm{d}$, the apparent crater radius $\mathrm{R}$, the soil density $\rho$, and two strength parameters to define the soil properties: one with the dimensions of a stress $\sigma$, related to soil strength, and other with the dimensions of a force divided by a cubic length $\left(\mathrm{Nm}^{-3}\right) \mathrm{K}$, that takes into account gravitational effects.

After a dimensional analysis and many empirical observations, the following functional relation may be obtained (Baker et al. 1991)

$$
\frac{R}{d}=f\left(\frac{W^{7 / 24}}{\sigma^{1 / 6} K^{1 / 8} d}\right)
$$

If $\frac{R}{d}$ (scaled radius of the crater) is plotted as a function of $W^{7 / 24} / d$, it can be seen that this relation is close to experimental results and can be approximately simplified by two straight lines, one with a moderate slope for $W^{7 / 24} / d>0.3$ and one steeper for $W^{7 / 24} / d<0.3$. For $W^{7 / 24} / d<0.3$, the scaled radius of the crater is sensible to small changes in the independent parameter and, due to this fact, the independent parameter or the scaled radius may exhibit great variability. Experimental conditions are better controlled for $W^{7 / 24} / d>0.3$. 
It can be deduced that the specific weight $\rho g$ is the best measure for $\mathrm{K}$ and that $\rho c^{2}$ is the best measure for $\sigma$, where $\mathrm{c}$ is the seismic velocity in the soil. If experimental results for different types of soils are plotted in a $\frac{R}{d}$ versus $\frac{W^{7 / 24}}{\rho^{7 / 24} c^{1 / 3} g^{1 / 8} d}$ graph, it may be clearly seen that there is very little variability in the results.

The numerical evaluation of the dimensions of craters produced by underground cylindrical TNT loads is presented in this paper. Although, there are many experimental results and empirical equations for the size of craters produced by underground explosions, the main objective of this paper is to check the ability of numerical tools to reproduce experimental values in order to use these tools for more complex problems such as the evaluation of damage produced on vehicles or objects situated on the ground by underground explosions.

The analysis is performed with a hydrocode (AUTODYN v6.1). Many alternative models for the same problem are first run in order to check the variability of the results. Then the dimensions of the craters for different TNT masses and depths are obtained and compared with experimental values.

The crater dimensions defined by Kinney and Graham (1985) are used in this work (Figure $1)$ : $D$ is the apparent crater diameter, $D_{r}$ is the actual crater diameter and $\mathrm{H}_{2}$ is the apparent depth of the crater.

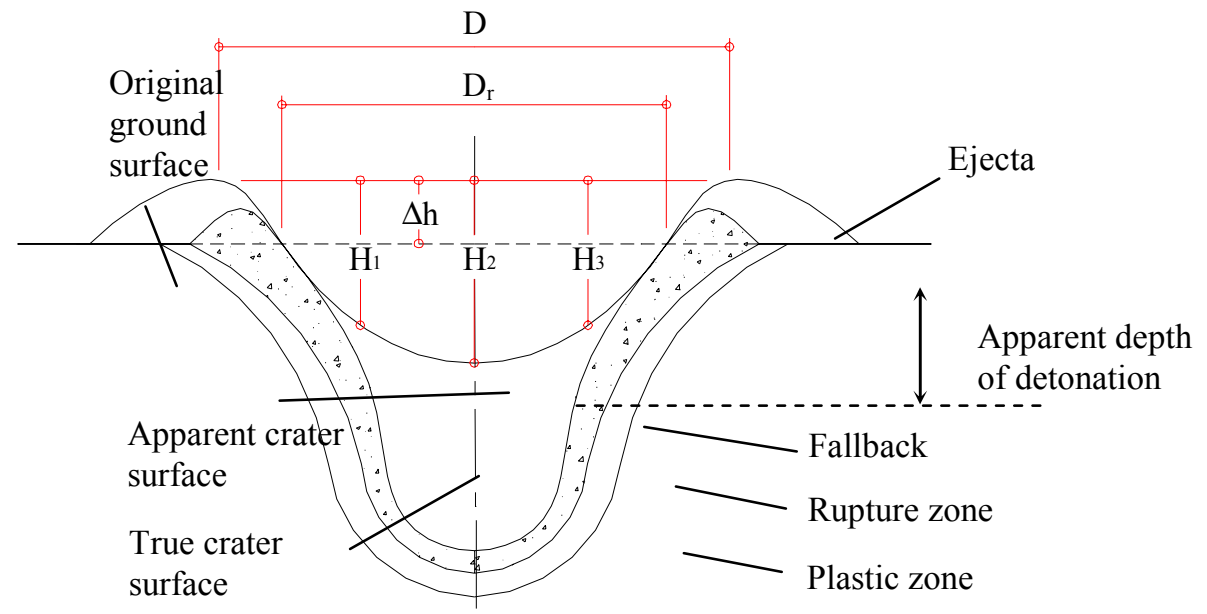

Figure 1: Definitions of the crater dimensions

\section{PROBLEM DESCRIPTION}

\subsection{Introduction}

First a typical problem is chosen in order to study the variability obtained in craters dimensions when different processors, different mesh sizes and slightly different physical models are used. Once these aspects have been checked, the mass and depth of the explosive are varied, maintaining all the other properties, and the resulting craters dimensions are compared with experimental and empirical values.

\subsection{Explosive}

In order to carry out a comparable analysis, the mass of the explosive is defined by TNT masses. The corresponding masses for other explosives can be obtained through the concept of TNT equivalence (Formby, 1996). 
A mass of $8 \mathrm{~kg}$ of TNT is defined for the typical problem and then the mass is varied from 0.26 to $8 \mathrm{~kg}$ of TNT.

A cylindrical explosive load like that represented in Figure 2 is considered for all the problems. The dimensions of the TNT load for the typical problem are also indicated in Figure 2. For the other problems the TNT mass is varied preserving the shape and the aspect ratio of the explosive load.

The explosive charge includes a Pentolite booster with a hole for the detonator in the centre of the TNT cylindrical charge.

\subsection{Soil}

All the results are obtained for a ferricrete, gravelly sand soil with the following properties, Dry Density at $150 \mathrm{~mm}: 1987 \mathrm{~kg} / \mathrm{m}^{3}$, at $300 \mathrm{~mm} 2071 \mathrm{~kg} / \mathrm{m}^{3}$, PI: 4 and 5 .

\subsection{Location}

For the typical problem the cylindrical charge is buried with top surface $50 \mathrm{~mm}$ below the soil level. The charge is covered up to the ground level with loose soil (no strength). The setup is graphically displayed in Figure 2.

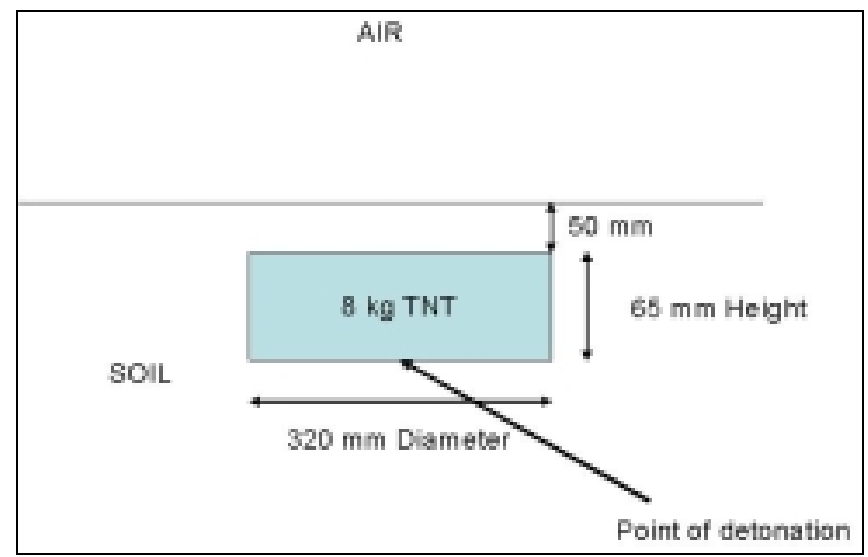

Figure 2: Typical problem set-up

Problems varying the depth of the explosive load were also studied.

The detonation point is considered to be placed in the center of the lower face of the explosive load as indicated in Figure 2. Nevertheless, the variation of results for other positions of the detonation point is also studied.

\section{NUMERICAL MODELS}

\subsection{Introduction}

Many alternative numerical models for the same physical problem were run, analyzed and compared. The alternatives that gave comparable and more reliable results are described in the following sections.

First, the effect of the size and refinement of the numerical mesh was studied. Different meshes were analyzed form a fine mesh with 332500 to the coarsest mesh with 67200 cells. 
Based on the convergence of results, the mesh presented in Section 3.2 was adopted.

Different numerical processors, Euler and Lagrange, were used to model the soil. The models and results obtained are presented in Sections 3.2 and 4 respectively.

Moreover, different alternatives for the constitutive model of the soil were used.

\subsection{Numerical mesh and processors}

The symmetry conditions allow using a two-dimensional (2D) mesh considering axial symmetry. A $13 \mathrm{~m} \times 4 \mathrm{~m}$ mesh with a minimum $10 \mathrm{~mm} \times 10 \mathrm{~mm}$ size was used. This mesh represents an $8 \mathrm{~m}$-diameter cylinder.

The mesh used for the typical problem is shown in Figure 3a. The mesh was filled with different materials: air, TNT and soil, indicated with different colors in Figure $3 b$.

A previous estimation of the final diameter and depth of the crater has been made and the mesh was refined with $1 \mathrm{~cm}$ cell all around the estimated final crater. Then, the maximum error in the final diameter of the crater is $2 \mathrm{~cm}$ and the maximum error in the depth is $1 \mathrm{~cm}$.

The following two alternative models using different numerical processors for the soil were compared.

a) In this model an Euler Godunov multi material with strength higher order processor is used to model the complete problem including the air, the explosive charge and the soil.

b) In this model an Euler Godunov processor is used to model the air and the explosive charge while a Lagrange processor is used for the soil. Coupling between Euler and Lagrange processors together with an erosion algorithm for the soil were defined in this case.

\subsection{Material models}

\subsubsection{Air}

The ideal gas equation of state was used for the air. This is one of the simplest forms of equation of state for gases. In an ideal gas, the internal energy is a function of the temperature alone and if the gas is polytropic the internal energy is simply proportional to temperature. It follows that the equation of state for a gas, which has uniform initial conditions, may be written as,

$$
p=(\gamma-1) \rho e
$$

in which $\mathrm{p}$ is the hydrostatic pressure, $\rho$ is the density and e is the specific internal energy. $\gamma$ is the adiabatic exponent, it is a constant (equal to $1+\mathrm{R} / \mathrm{cv}$ ) where constant $\mathrm{R}$ may be taken to be the universal gas constant R0 divided by the effective molecular weight of the particular gas and cv is the specific heat at constant volume. The values of the constants used for air are presented in Table 1.

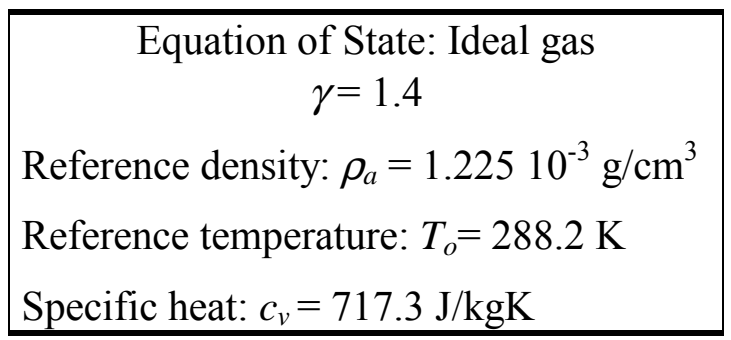

Table 1: Air properties 
AUTODYN-2D v6. 1 from Century Dynamics

Gycle 0

Time 0.000E+000 ms

Units $\mathrm{mm}, \mathrm{mg}, \mathrm{ms}$

Axial symmetry

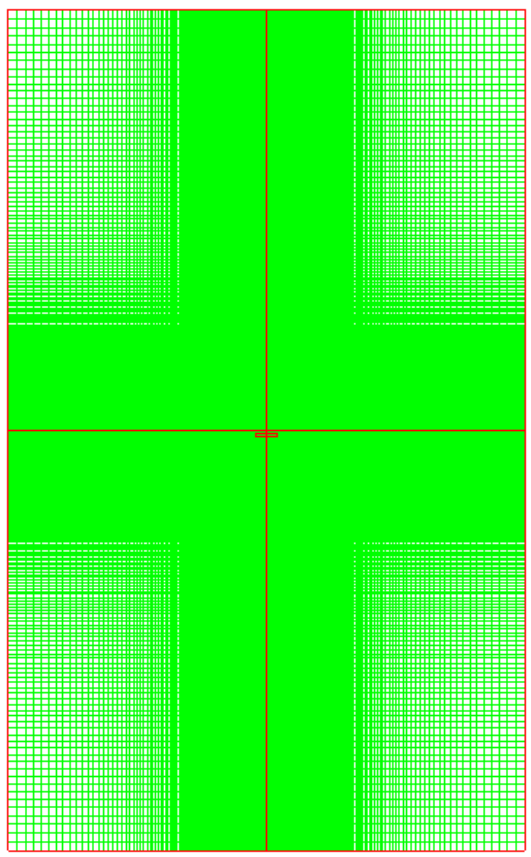

(a)

AN TODYN-2D v6. 1 from Century Dynamics

Material Location

Void

AIR

TNT

suelo
Cycle 0

Time $0.000 E+000 \mathrm{~ms}$

Units $\mathrm{mm}, \mathrm{mg}, \mathrm{ms}$

Axial symmetry

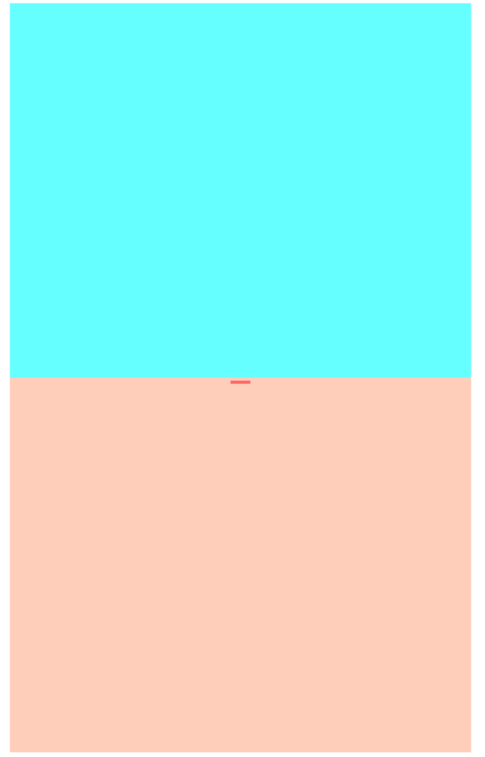

(b)

Figure 3: Numerical model. a) Mesh b) Materials 


\subsubsection{TNT}

An explosion may be initiated by various methods. However, initiation of an explosive always goes through a stage in which a shock wave is an important feature. Lee-Tarver equation of state (Lee and Tarver, 1980) was used to model both the detonation and expansion of TNT in conjunction with "Jones - Wilkins - Lee" (JWL EOS) to model the unreacted explosive.

The (JWL) equation of state can be written as,

$$
p=C_{1}\left(1-\frac{\omega}{r_{1} v}\right) e^{-r_{1} v}+C_{2}\left(1-\frac{\omega}{r_{2} v}\right) e^{-r_{2} v}+\frac{\omega e}{v}
$$

Where $v=1 / \rho$ is the specific volume, $\mathrm{C}_{1}, \mathrm{r}_{1}, \mathrm{C}_{2}, \mathrm{r}_{2}$ and $\omega$ (adiabatic constant) are constants and their values have been determined from dynamic experiments and are available in the literature for many common explosives. The values used for TNT are presented in Table 2.

\begin{tabular}{|l|}
\hline \multicolumn{1}{|c|}{ Equation of State: JWL } \\
Reference density $\rho=1.658 \mathrm{~g} / \mathrm{cm}^{3}$ \\
$C_{1}=3.737710^{8} \mathrm{kPa}$ \\
$C_{2}=3.7347110^{6} \mathrm{kPa}$ \\
$R_{1}=4.15$ \\
$R_{2}=0.9$ \\
$\omega=0.35$ \\
C-J detonation velocity: $6.9310^{3} \mathrm{~m} / \mathrm{s}$ \\
C-J energy $/$ unit volumen: $610^{6} \mathrm{KJ} / \mathrm{m}^{3}$ \\
C-J pressure: $2.110^{7} \mathrm{kPa}$ \\
\hline
\end{tabular}

Table 2: TNT properties

\subsubsection{Soil}

A shock equation of state combined with an elastoplastic strength model based on Drucker Prager criterion and a hydro tensile limit were used for the soil. The initial density was taken as

$\rho=2.2 \mathrm{~g} / \mathrm{cm} 3$ (wet density). The wet density was obtained considering a mean dry density of $2100 \mathrm{~kg} / \mathrm{m} 3$ and a moisture content of $5 \%$.

For non-cohesive soils, it could be necessary to use a shear modulus varying with depth. In absence of SPT data or other useful data such us Dynamic Cone Penetration Tests or Field Vane Test, a medium shear modulus in all depth was used.

A Drucker Prager criterion with standard values was adopted for the strength model. A summary of soil properties used for all the models is presented in Table 3

It is important to note that it was previously proved (Ambrosini et al. 2004) that the 
influence of the soil properties on the size of craters produced by explosive loads is usually rather small (variations of about $\pm 5 \%$ in crater diameter could be obtained).

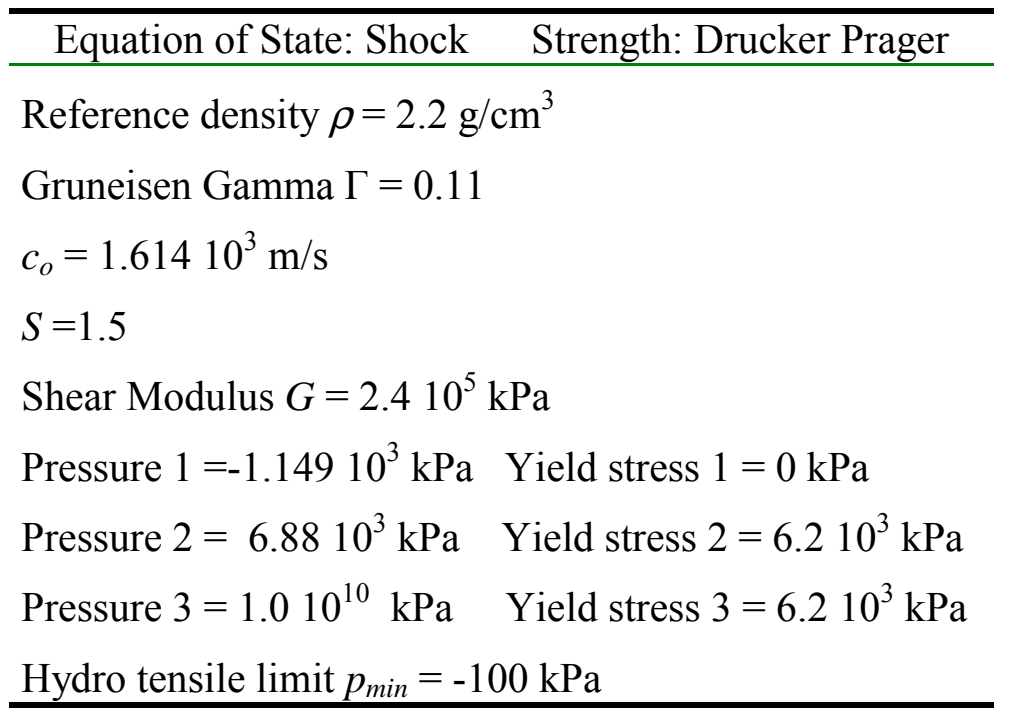

Table 3: Soil properties

Many alternatives for the loose soil over the explosive charge were simulated: a soil with the same properties of the rest of the soil, a soil with reduced strength and stiffness (one half mass density and an order of magnitude minor shear elasticity modulus were used) and non soil but air over the explosive charge

\section{4 . Boundary transmit}

In order to fulfill the radiation condition, a transmitting boundary was defined for air as well as soil subgrids external limits. The transmit boundary condition allows a stress wave to continue "through" the physical boundary of the subgrid without reflection. The size of the numerical mesh can be reduced by use of this boundary condition. The transmit boundary is only active for flow out of a grid.

\section{NUMERICAL RESULTS FOR THE TYPICAL PROBLEM}

First, the results obtained for both models described for the typical problem are presented. According to a previous work (Ambrosini and Luccioni, 2006), the mechanical properties of the soil do not significantly affect the diameter of the crater obtained. However, a variation of $\pm 5 \%$ could be obtained in the crater diameter.

A larger model than those found in other papers and works in the literature was used. This model allows checking when the explosive wave is far away from both the air and the soil. The problem has been run until the crater dimensions remained stable.

In other works, a time of approximately $20 \mathrm{~ms}$ from the detonation instant has been determined as enough to obtain the final dimensions of the crater (Ambrosini et al. 2003; Ambrosini et al. 2004; Ambrosini and Luccioni, 2006).

\subsection{Euler processor. Model a.}

The final state of the model is presented in Figure 4. 


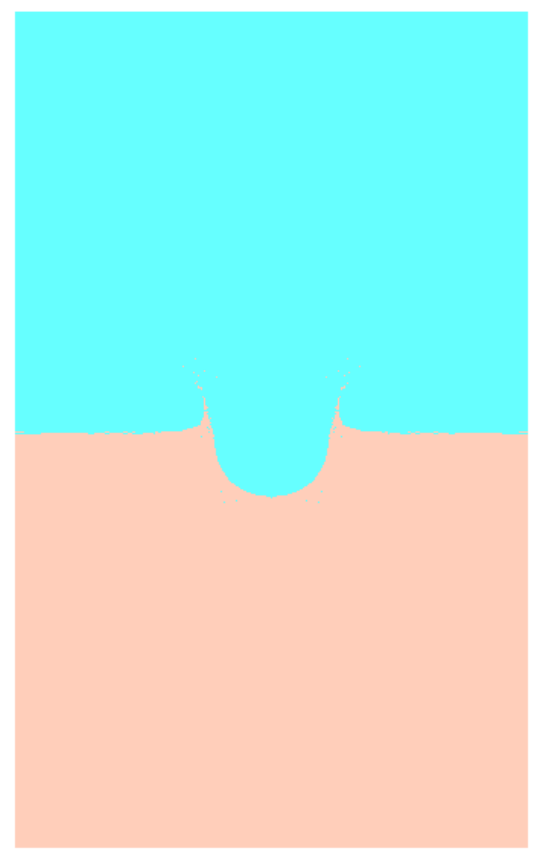

Figure 4. Crater obtained with an Euler processor for the soil

The apparent diameter of the crater obtained is $D=2.07 \mathrm{~m}$

\subsection{Lagrange processor. Model b.}

The final state of the model is presented in Figure 5.

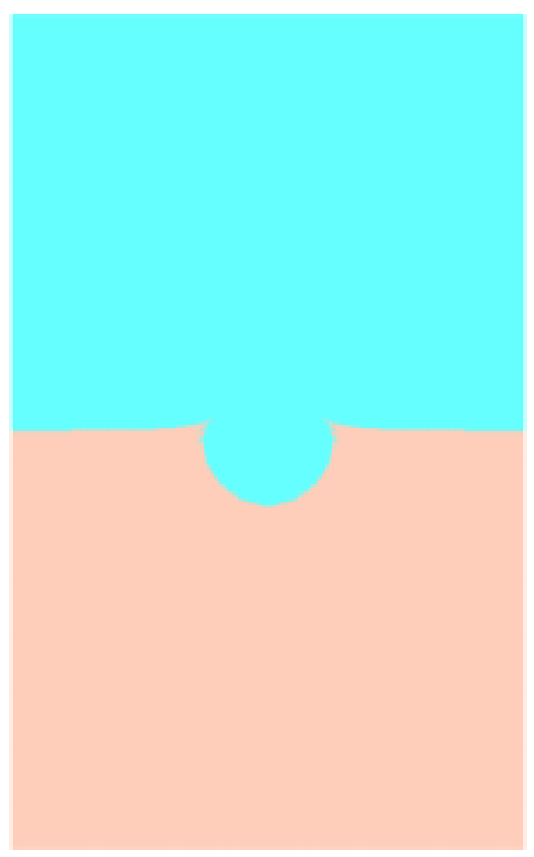

Figure 5. Crater obtained with a Lagrange processor for the soil

The apparent diameter of the crater obtained $D=2.06 \mathrm{~m}$ 


\subsection{Other alternatives simulated.}

The results obtained for the crater diameter of the typical problem, varying the properties of the soil and the position of the detonation point are coincident with those presented in previous sections.

\section{VARIATION OF CRATER DIAMETER WITH SCALED DISTANCE}

The diameter of the craters produced by different TNT masses located different depths are obtained in this section and compared with experimental results. The results for different scaled distances are presented in Table 4 and Figure 6 together with the experimental results presented by Baker et al. (1991) for alluvium soils and results presented by Ambrosini et al.(2002) for spherical underground explosions in reddish brown clayey silt of low plasticity.

The numerical results were obtained with a model similar to model a, using an Euler processor. A cylindrical explosive load of 0.26 or $8 \mathrm{~kg}$ of TNT was used and depth was varied. The mesh was always refined in the surroundings of the explosive charge.

Although the types of soils involved and the shape of the explosive load are different, an excellent agreement between the present numerical results and those corresponding to Baker et al.(1991) and Ambrosini et al.(2002) can be observed in Figure 6.

\begin{tabular}{|c|c|c|c|}
\hline $\begin{array}{c}\text { Mass of Explosive } \\
\mathrm{W}(\mathrm{kg} \text { of TNT) }\end{array}$ & $\begin{array}{c}\text { Depth } \\
\mathrm{d}(\mathrm{m})\end{array}$ & $\begin{array}{c}W^{7 / 24} / \mathrm{d} \\
\left(\mathrm{kg}^{7 / 24} / \mathrm{m}\right)\end{array}$ & $\begin{array}{c}\text { Crater diameter } \\
\mathrm{D}(\mathrm{m})\end{array}$ \\
\hline 8 & 0.032 & 0.48 & 2.02 \\
\hline 8 & 0.082 & 0.67 & 2.07 \\
\hline 8 & 0.20 & 1.12 & 2.60 \\
\hline 8 & 0.50 & 4.00 & 3.12 \\
\hline 0.26 & 0.60 & 10.00 & 1.27 \\
\hline 0.26 & 1.00 & 22.23 & 0.92 \\
\hline 0.26 & 1.40 & 61.5 & 0.70 \\
\hline
\end{tabular}

Table 3: Crater diameter for different TNT masses and depths. 


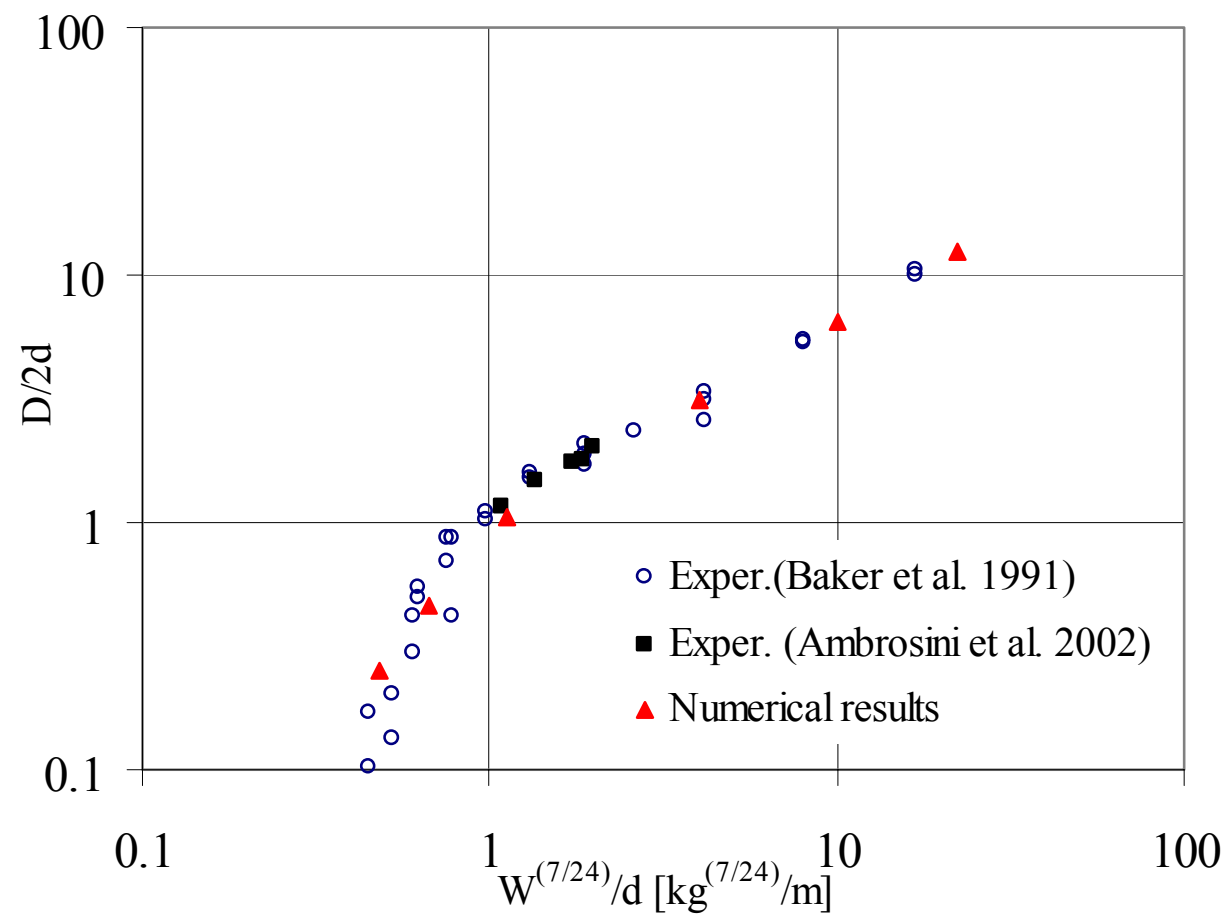

Figure 6. Variation of crater diameter with scaled distance. Comparison with experimental results

\section{CONCLUSIONS}

A very good agreement is observed between the crater diameter obtained with an Euler processor for the soil (model a) and that obtained with a Lagrange processor (model b). The crater obtained with model $b$ is deeper than that obtained with model a. In both cases, it is clear that the obtained depth is greater than the apparent depth observed in an experimental test (see Figure 1). The final shape of the crater obtained with model a better resembles the actual one than that obtained with model $b$.

It has been proved that the effect of the loose soil above the explosive and the position of the pentolite booster practically do not influence the final crater diameter.

A good agreement in crater diameter with experimental results obtained by other researchers and the authors has been obtained. The shape of the explosive load and the type has practically non influence in the value of the crater diameter.

It is important to note that the software used gives the possibility of finding the rupture and plastic zones of the crater. In order to use the results given by the software related to rupture and plastic zones, a more detailed study of the soil model and soil properties used should be carried out, because they will obviously influence those results. Erosion limit used in Lagrange simulations has been found to be an important point to study because not only the dimensions of the rupture zone but also the stability of the numerical solution strongly depends on this value. The influence of the erosion limit value in relation to the Lagrange mesh size was not studied but could be subject of future research. 


\section{AKNOWLEDGEMENTS}

The authors wish to thank the help received from Mrs. Amelia Campos for the English revision. The financial support of the CONICET (Argentina) and CIUNT )National University of Tucumán) is gratefully acknowledged.

\section{REFERENCES}

R.D. Ambrosini, RD., B. Luccioni, R. Danesi, J. Riera and M. Rocha. Size of Craters Produced by Explosive Charges on or Above the Ground Surface. Shock Waves, 12(1):6978, 2002.

R.D. Ambrosini, B. Luccioni and R. Danesi. Craters produced by explosions on the soil surface. Computational Mechanics, XXII:678-692, 2003.

R.D. Ambrosini, B. Luccioni and R. Danesi. Influence of the soil properties on craters produced by explosions on the soil surface. Computational Mechanics, XXIII:571-590, 2004.

R.D. Ambrosini and B. Luccioni. Craters produced by explosions on the soil surface. Journal of Applied Mechanics, ASME, in press, 2006

AUTODYN, Explicit Software for Non-Linear Dynamics, Version 6.1, User's Manual. Century Dynamics Inc, 2005.

W.E. Baker, P.S. Westine and F.T. Dodge FT. Similarity methods in engineering dynamics. Elsevier, Amsterdam, 1991

J.W. Bull, C.H.Woodford. Camouflets and their effects on runway supports. Computer and Structures, 69(6):695-706, 1998.

S.A. Formby and R.K. Wharton. Blast characteristics and TNT equivalence values for some commercial explosives detonated at ground level. Journal of Hazardous Materials, 50:183$198,1996$.

G. F. Kinney and K.J. Graham. Explosive shocks in air. 2nd Edition, Springer Verlag, 1985.

E.L. Lee and C.M. Tarver. Phenomenological model of shock initiation in heterogeneous explosives. Physics of Fluids, 23(12):2362-2372, 1980.

P.A. Persson, R. Holmberg and J. Lee J. Rock blasting and explosives engineering. CRC Press, USA, 1994. 